Journal of Engineering Sciences, Assiut University, Vol. 35, No. 2, pp. 323-335, March 2007

\title{
A MATHEMATICAL EXPRESSION FOR SPLIT TENSILE STRENGTH OF STEEL FIBER REINFORCED CONCRETE
}

\author{
Mazen A. Musmar ${ }^{1}$ and Muhammad I. Rjoub ${ }^{2}$ \\ Civil Engineering Department, Faculty of Engineering Technology, Al-Balqa' \\ Applied University. Amman 11134, P.O. Box 15008, Jordan \\ ${ }^{1}$ Email:m_musmar@wanadoo.jo
}

(Received November 11, 2006 Accepted February 5, 2007)

\begin{abstract}
Use of fiber reinforced concrete is increasing recently, brought forth by the enhancements of concrete matrix engineering properties upon adding fiber. The resulting material possesses higher tensile strength, consolidated response and better ductility, which ultimately minimize immature failures.

Thus it is beneficial to comprehend more the engineering properties of the material. Accordingly, this study moves toward deriving a mathematical expression that relates fiber reinforced concrete compressive strength to split cylinder tensile strength.

Literature survey is carried out to collect data, pertinent to split cylinder tensile strength versus compressive strength values, for fiber reinforced concrete. Gathered data comprise compressive strengths from $20 \mathrm{MPa}$ to $102 \mathrm{MPa}$.

Regression analysis is performed; a mathematical expression that predicts split cylinder tensile strength of steel fiber reinforced concrete is eventually derived. The predicted values fit well with experimental data. The findings of this study shed more light on the tensile strength of fiber reinforced concrete.
\end{abstract}

KEYWORDS: Steel Fiber Reinforced concrete, composite concrete

\section{1- INTRODUCTION}

The addition of steel fibers to concrete mixes improves the properties of the obtained steel fiber reinforced concrete (SFRC). Symptoms of the improved properties comprise tensile strength, shear strength as well as ductility and toughness of the SFRC.

Such enhancements render using steel fibers attractive especially in high strength concrete. The addition of steel fibers also reduces the concrete brittleness, which increases with higher concrete strengths. Such improvements in concrete properties support the use of SFRC in many structural applications such as airports, highway paving layers, industrial floorings, and bridge decks. Let alone its use as a repairing material. 
Utilizing steel fibers as a technique for improving the properties of concrete has been the issue of many studies on structural behavior of such material, steered to acquire better understanding of the behavior of SFRC both in linear and nonlinear stages, and to attain detailed authenticated provisions for the design of such materials.

Few studies have been targeted towards investigating the relationship between the split tensile strength and the compressive strength of SFRC. The available relationships are either based on limited number of specimens or narrow range of fiber content or fiber aspect ratio. Ashour et al. [1] suggested the following equation for high strength concrete specimens of a single aspect ratio, $l / d$ of 75

$$
f_{s p}=4.95-2.13 v_{f}
$$

where $v_{f}$ is fiber content.

More parameters are presented within the expression addressed by Ashour et al. [2], as follows:

$$
f_{s p}=\frac{f_{c u f}}{(20-\sqrt{F})}+0.7+\sqrt{F}
$$

where $f_{\text {cuf }}$ is the cube strength of fiber reinforced concrete [MPa].

$f_{s p} \quad$ is the splitting strength of fiber reinforced concrete [MPa].

$F \quad$ is fiber reinforcement index $=(l / d) . v_{f} . D_{f}$, where $l$ and $d$ are steel fiber length and diameter respectively. $D_{f}$ is a bond factor that equals 0.5 for round fibers, 0.75 for crimped fibers, and 1.0 for indented fibers.

In order to derive a credible mathematical relationship, based on a wide range of fiber contents $\left(v_{f}\right)$, compressive strengths, and fiber aspect ratios $(l / d)$, this study is carried out; it embodies a large number of experimental data collected from literature. The independent predictors comprise specimens of different sizes, various concrete grades and aggregate types in addition to multiple values of the fiber reinforcement index $\left(v_{f} * \frac{l}{d}\right)$.

\section{2- RESEARCH SIGNIFICANCE}

The importance of the study is that it employs a large number of experimental data of SFRC, based on test specimens that cover a variety of factors of significant effect on the SFRC split strength. The main goal is to present a reliable mathematical expression that relates SFRC split strength with concrete cylindrical compressive strength and fiber reinforcement index. It is hoped that 
such expression could be ultimately employed in formulating appropriate design provisions for fibrous concrete members, under shear, torsion and tension.

\section{3- DATA ANALYSIS}

An experimental data pertinent to values of compressive strength $f_{c}{ }_{c}$, splitting tensile strength, $f_{s p}$, volumetric fiber content, $v_{f}$, and fiber aspect ratio, $l / d$ of 358 SFRC cylindrical specimens are listed in Table (3). These data are gathered from several research papers, (Batson [3], Craig et al. [4], Sharma [5], Robert and Victor [6], El-Niema [7], Ashour et al. [2], Ashour and Wafa [8], Ghosheh [9], Padmarajaiah [10], Marar and Celik [11], Kwak [12], Ayish [13], BaniYasin [14], Rjoub and Rasheed [15], and Aqaileh [16] ). Gathered data contain compressive strength values from 20.65 MPa to $102 \mathrm{MPa}$. Also data include concrete without fibers and concrete with fiber reinforcement indices. All the compressive strength values presented in Table (3) are either for cylinders of standard dimensions $(150 \times 300 \mathrm{~mm})$ or converted to standard cylindrical strengths using conversion factors presented in Table (2). Regression analysis is carried out with the split strength, $f_{s p}$ as the predicted dependent variable. The scatter plot of experimental values of $f_{s p}$ versus $f_{c}^{\prime}$ indicates that the expected relation could take the general expression

$$
f_{s p}=\alpha+\beta \times\left(\left(v_{f} \times \frac{l}{d}\right)^{\chi}\right) \times \sqrt{f_{c}^{\prime}}
$$

Parameters that are statistically insignificant are discarded, and the model coefficients are eventually determined. The values of calculated regression coefficients $(\alpha, \beta$ and $\chi)$ are found to be $(0.614,0.4$ and 1.029) respectively. Ultimately, the mathematical expression that predicts split cylinder tensile strength of fiber reinforced concrete $f_{s p}$ is concluded as follows

$$
f_{s p}=\left(0.614+0.4\left(v_{f} \times \frac{l}{d}\right)^{1.029}\right) \times \sqrt{f^{\prime}}
$$

The P-values for the coefficients of regression analysis $(\alpha, \beta$ and $\chi)$ are illustrated in Table (1).Their values are less than 0.001. Such low p-values indicate that the predictor has a significant effect on the response variable. Thus the predictors illustrated in Eq. (4) are statically significant, and have a definite significant effect on the predicted values. Also, the adjusted coefficient of determination, $\mathrm{R}^{2}$ is 0.840 , implying that the regression predicted values are acceptably close to the observed data. 
Equation (4) can be normalized by dividing its two sides by the term $\sqrt{f_{c}^{\prime}}$ as follows

$$
\frac{f_{s p}}{\sqrt{f^{\prime}{ }_{c}}}=\left(0.614+0.4 \quad\left(v_{f} \times \frac{l}{d}\right)^{1.029}\right)
$$

Equation (5) could be further simplified as follows

$$
\frac{f_{s p}}{\sqrt{f^{\prime}{ }_{c}}}=(0.6+0.4 \times(\% F R I))
$$

Where $F R I=v_{f} . l / d$

Figure (1) illustrates the scatter plot of \%FRI versus the experimental split strength divided by $\sqrt{f_{c}^{\prime}}$, for the data listed in Table (3). The plot illustrates an upper and lower bounds derived by regression analysis. Figure (2) also illustrates the experimental split strength values versus the predicted values according to Eq. (7). It indicates that the predicted values are close to test result values. The plot of the data in both figures (Figs. 1 and 2) confirms the reliability of the derived expression. Equation (6) may be written in the following form

$$
f_{s p}=\left(0.6+0.4 \quad\left(v_{f} \times \frac{l}{d}\right)\right) \times \sqrt{f^{\prime}}
$$

Table 1: Estimated parameters using regression analysis

\begin{tabular}{|c|c|c|c|c|c|}
\hline & $\alpha=0.614$ & $\beta=0.4$ & $\chi=1.029$ & $\mathrm{R}^{2}=0.841$ & adjusted $\mathrm{R}^{2}=0.840$ \\
\hline P value & $<0.001$ & $<0.001$ & $<0.001$ & & \\
\hline
\end{tabular}

Table 2: Conversion factors to standard cylindrical strength [15]

\begin{tabular}{|c|c|c|c|c|c|}
\hline $\begin{array}{c}\text { Cylinders } \\
7.5 \times 150 \mathrm{~mm}\end{array}$ & 0.95 & $\begin{array}{c}\text { Cylinders } \\
100 \times 200 \mathrm{~mm}\end{array}$ & 0.97 & $\begin{array}{c}\text { cylinders } \\
150 \times 300 \mathrm{~mm}\end{array}$ & 1 \\
\hline $\begin{array}{c}\text { Cubes } \\
100 \times 100 \times 100 \mathrm{~mm}\end{array}$ & 0.78 & $\begin{array}{c}\text { Cubes } \\
150 \times 150 \times 150 \mathrm{~mm}\end{array}$ & 0.8 & $\begin{array}{c}\text { Cubes } \\
200 \times 200 \times 200 \mathrm{~mm}\end{array}$ & 0.83 \\
\hline
\end{tabular}


Table 3: Compressive strength, fiber reinforcement index and split cylinder strength

\begin{tabular}{|c|c|c|c|c|c|c|c|}
\hline \multicolumn{8}{|c|}{$\begin{array}{l}\text { Marar and Celic [11] } \\
\text { (Compression, splitting, Cylinders 150x300) }\end{array}$} \\
\hline no & FRI & $\mathrm{f}^{\prime} \mathrm{c}(\mathrm{Mpa})$ & $\begin{array}{c}\mathrm{f}_{\mathrm{sp}} \\
(\mathrm{MPa})\end{array}$ & no & FRI & $\mathrm{f}^{\prime} \mathrm{c}(\mathrm{Mpa})$ & $f_{s p}(M P a)$ \\
\hline 1 & 0 & 32.06 & 3.2 & 20 & 0 & 73.5 & 5.13 \\
\hline 2 & 30 & 32.66 & 3.93 & 21 & 30 & 76.02 & 5.68 \\
\hline 3 & 60 & 34.11 & 4.72 & 22 & 60 & 78.48 & 6.95 \\
\hline 4 & 75 & 36.28 & 5.35 & 23 & 75 & 80.09 & 8.26 \\
\hline 5 & 90 & 37.46 & 5.9 & 24 & 90 & 84.63 & 8.93 \\
\hline 6 & 105 & 39.27 & 6.1 & 25 & 105 & 86.22 & 9.97 \\
\hline 7 & 120 & 39.85 & 6.84 & 26 & 120 & 88.97 & 10.83 \\
\hline 8 & 37.5 & 33.73 & 4.12 & 27 & 37.5 & 76.96 & 6.94 \\
\hline 9 & 75 & 34.63 & 5.24 & 28 & 75 & 78.85 & 8.14 \\
\hline 10 & 93.75 & 36.61 & 6.18 & 29 & 93.75 & 84.48 & 9.12 \\
\hline 11 & 112.5 & 38.31 & 6.53 & 30 & 112.5 & 87.4 & 10.03 \\
\hline 12 & 131.25 & 39.63 & 7.15 & 31 & 131.25 & 89.52 & 11.16 \\
\hline 13 & 150 & 41.17 & 7.87 & 32 & 150 & 91.49 & 11.74 \\
\hline 14 & 41.5 & 33.99 & 4.36 & 33 & 41.5 & 78.02 & 7.51 \\
\hline 15 & 83 & 35.26 & 5.94 & 34 & 83 & 80.95 & 8.89 \\
\hline 16 & 103.75 & 37.09 & 6.54 & 35 & 103.75 & 86.21 & 10.71 \\
\hline 17 & 124.5 & 39.73 & 7.07 & 36 & 124.5 & 89.19 & 11.5 \\
\hline 18 & 145.25 & 41.27 & 7.86 & 37 & 145.25 & 91.73 & 12.54 \\
\hline 19 & 166 & 42.87 & 8.33 & 38 & 166 & 93.56 & 13.16 \\
\hline \multicolumn{8}{|c|}{ Craig et al [4] } \\
\hline no & FRI & $\mathrm{f}^{\prime} \mathrm{c}(\mathrm{Mpa})$ & $\begin{array}{c}\mathrm{f}_{\mathrm{sp}} \\
(\mathrm{MPa})\end{array}$ & no & FRI & $\mathrm{f}^{\prime} \mathrm{c}(\mathrm{Mpa})$ & $\mathrm{f}_{\mathrm{sp}}(\mathrm{MPa})$ \\
\hline 39 & 0.00 & 40.69 & 3.45 & 43 & 120.00 & 28.97 & 4.55 \\
\hline 40 & 42.00 & 40.00 & 5.72 & 44 & 200.00 & 47.59 & 6.00 \\
\hline 41 & 100.00 & 43.45 & 6.34 & 45 & 120.00 & 40.00 & 6.07 \\
\hline 42 & 90.00 & 35.86 & 5.31 & 46 & 160.00 & 45.52 & 7.10 \\
\hline \multicolumn{8}{|c|}{ Sharma [5] } \\
\hline no & FRI & $\mathrm{f}^{\prime} \mathrm{c}(\mathrm{Mpa})$ & $\begin{array}{c}\mathrm{f}_{\mathrm{sp}} \\
(\mathrm{MPa})\end{array}$ & no & FRI & $\mathrm{f}^{\prime} \mathrm{c}(\mathrm{Mpa})$ & $\mathrm{f}_{\mathrm{sp}}(\mathrm{MPa})$ \\
\hline 47 & 0 & 42.3 & 4.55 & 51 & 72 & 48.6 & 7.16 \\
\hline 48 & 0 & 43.2 & 4.6 & 52 & 67.5 & 47.7 & 6.96 \\
\hline 49 & 0 & 47.7 & 4.83 & 53 & 67.5 & 43.2 & 6.62 \\
\hline 50 & 0 & 46.8 & 4.79 & & & & \\
\hline
\end{tabular}




\begin{tabular}{|c|c|c|c|c|c|c|c|}
\hline \multicolumn{8}{|c|}{ Batson [3] } \\
\hline no & FRI & $\begin{array}{c}\mathrm{f}^{\prime} \mathrm{c} \\
(\mathrm{Mpa})\end{array}$ & $\begin{array}{c}\mathrm{f}_{\mathrm{sp}} \\
(\mathrm{MPa})\end{array}$ & no & FRI & $\mathrm{f}^{\prime} \mathrm{c}(\mathrm{Mpa})$ & $\mathrm{f}_{\mathrm{sp}}(\mathrm{MPa})$ \\
\hline 54 & 44 & 40.19 & 5.71 & 63 & 61.6 & 39.71 & 6.18 \\
\hline 55 & 44 & 40.19 & 5.71 & 64 & 61.6 & 39.71 & 6.18 \\
\hline 56 & 44 & 40.19 & 5.71 & 65 & 61.6 & 39.71 & 6.18 \\
\hline 57 & 44 & 40.19 & 5.71 & 66 & 61.6 & 39.71 & 6.18 \\
\hline 58 & 30.8 & 40.19 & 5.71 & 67 & 61.6 & 39.71 & 6.18 \\
\hline 59 & 30.8 & 40.19 & 5.71 & 68 & 61.6 & 39.71 & 6.18 \\
\hline 60 & 30.8 & 40.19 & 5.71 & 69 & 61.6 & 39.71 & 6.18 \\
\hline 61 & 30.8 & 40.19 & 5.71 & 70 & 61.6 & 39.71 & 6.18 \\
\hline 62 & 61.6 & 39.71 & 6.18 & & & & \\
\hline \multicolumn{8}{|c|}{ Ghosheh [9] } \\
\hline no & FRI & $\mathrm{f}^{\prime} \mathrm{c}(\mathrm{Mpa})$ & $\begin{array}{c}\mathrm{f}_{\mathrm{sp}} \\
(\mathrm{MPa})\end{array}$ & no & FRI & $\mathrm{f}^{\prime} \mathrm{c}(\mathrm{Mpa})$ & $\mathrm{f}_{\mathrm{sp}}(\mathrm{Mpa})$ \\
\hline 71 & 0 & 42.49 & 4.56 & 78 & 75 & 42.67 & 5.69 \\
\hline 72 & 0 & 41.9 & 4.53 & 79 & 56.25 & 40.47 & 6.62 \\
\hline 73 & 0 & 41.9 & 4.53 & 80 & 93.75 & 40.85 & 6.11 \\
\hline 74 & 26.6 & 42.49 & 5.4 & 81 & 37.5 & 40.47 & 7.17 \\
\hline 75 & 35 & 39.7 & 5.49 & 82 & 75 & 40.11 & 5.51 \\
\hline 76 & 70 & 41.42 & 6.7 & 83 & 0 & 41.42 & 6.7 \\
\hline 77 & 37.5 & 40.11 & 5.24 & & & & \\
\hline \multicolumn{8}{|c|}{ Bani-Yasin [14] } \\
\hline no & FRI & $\mathrm{f}^{\prime} \mathrm{c}(\mathrm{Mpa})$ & $\begin{array}{c}\mathrm{f}_{\mathrm{sp}} \\
(\mathrm{Mpa})\end{array}$ & no & FRI & $\mathrm{f}^{\prime} \mathrm{c}(\mathrm{Mpa})$ & $\mathrm{f}_{\mathrm{sp}}(\mathrm{Mpa})$ \\
\hline 84 & 0.00 & 23.83 & 2.62 & 99 & 0.00 & 51.90 & 5.66 \\
\hline 85 & 0.00 & 23.57 & 2.49 & 100 & 0.00 & 49.64 & 5.23 \\
\hline 86 & 0.00 & 23.27 & 2.54 & 101 & 0.00 & 52.13 & 5.64 \\
\hline 87 & 0.00 & 24.20 & 2.49 & 102 & 0.00 & 53.80 & 5.73 \\
\hline 88 & 0.00 & 23.75 & 2.46 & 103 & 0.00 & 52.94 & 5.86 \\
\hline 89 & 0.00 & 23.82 & 2.51 & 104 & 0.00 & 52.89 & 5.94 \\
\hline 90 & 30.00 & 25.83 & 2.86 & 105 & 30.00 & 54.25 & 6.44 \\
\hline 91 & 30.00 & 24.66 & 3.02 & 106 & 30.00 & 55.54 & 6.12 \\
\hline 92 & 30.00 & 24.90 & 2.97 & 107 & 30.00 & 54.72 & 6.13 \\
\hline 93 & 60.00 & 27.39 & 3.44 & 108 & 60.00 & 55.72 & 7.42 \\
\hline 94 & 60.00 & 26.41 & 3.15 & 109 & 60.00 & 55.85 & 7.28 \\
\hline 95 & 60.00 & 25.99 & 3.01 & 110 & 60.00 & 56.80 & 7.29 \\
\hline 96 & 75.00 & 26.68 & 3.88 & 111 & 75.00 & 55.26 & 7.65 \\
\hline 97 & 75.00 & 25.63 & 3.64 & 112 & 75.00 & 55.88 & 7.55 \\
\hline 98 & 75.00 & 26.19 & 3.58 & 113 & 75.00 & 55.58 & 7.48 \\
\hline
\end{tabular}




\section{Ayish[13]}

\begin{tabular}{|c|c|c|c|c|c|c|c|}
\hline no & FRI & f'c $^{\prime}(\mathrm{Mpa})$ & $\mathrm{f}_{\mathrm{sp}}(\mathrm{Mpa})$ & no & FRI & $\mathrm{f}^{\prime} \mathrm{c}(\mathrm{Mpa})$ & $\mathrm{f}_{\mathrm{sp}}(\mathrm{Mpa})$ \\
\hline 114 & 0 & 20.1 & 3.1 & 119 & 60 & 22.78 & 2.94 \\
\hline 115 & 30 & 21.37 & 3.23 & 120 & 60 & 24.65 & 2.76 \\
\hline 116 & 60 & 22.753 & 3.67 & 121 & 60 & 23.02 & 2.79 \\
\hline 117 & 60 & 22.91 & 3.53 & 122 & 90 & 24.65 & 3.58 \\
\hline 118 & 0 & 20.65 & 3.14 & 123 & 90 & 25.45 & 3.63 \\
\hline 124 & 60 & 22.15 & 4.08 & 140 & 90 & 24.73 & 3.68 \\
\hline 125 & 0 & 32.76 & 3.84 & 141 & 30 & 53.52 & 5.96 \\
\hline 126 & 30 & 34.48 & 4.15 & 142 & 30 & 52.03 & 6.08 \\
\hline 127 & 60 & 35.72 & 4.66 & 143 & 30 & 53.66 & 5.91 \\
\hline 128 & 60 & 36.43 & 4.36 & 144 & 60 & 51.74 & 5.73 \\
\hline 129 & 0 & 32.60 & 3.74 & 145 & 60 & 52.74 & 5.86 \\
\hline 130 & 60 & 35.96 & 4.64 & 146 & 60 & 53.35 & 5.94 \\
\hline 131 & 30 & 21.73 & 2.48 & 147 & 30 & 54.52 & 6.26 \\
\hline 132 & 30 & 22.07 & 2.31 & 148 & 30 & 53.66 & 6.57 \\
\hline 133 & 30 & 21.55 & 2.47 & 149 & 30 & 54.29 & 6.45 \\
\hline 134 & 60 & 22.33 & 2.49 & 150 & 60 & 54.85 & 6.89 \\
\hline 135 & 60 & 22.08 & 2.46 & 151 & 60 & 56.68 & 7.3 \\
\hline 136 & 60 & 22.16 & 2.51 & 152 & 60 & 54.14 & 7.13 \\
\hline 137 & 30 & 22.72 & 2.6 & 153 & 90 & 56.64 & 8.56 \\
\hline 138 & 30 & 22.32 & 2.66 & 154 & 90 & 56.03 & 8.34 \\
\hline 139 & 30 & 22.25 & 2.54 & 155 & 90 & 56.72 & 8.63 \\
\hline
\end{tabular}

Kwak [12]

\begin{tabular}{|c|c|c|c|c|c|c|c|}
\hline no & FRI & $\mathrm{f}^{\prime} \mathrm{c}(\mathrm{Mpa})$ & $\mathrm{f}_{\mathrm{sp}}(\mathrm{Mpa})$ & no & FRI & $\mathrm{f}^{\prime} \mathrm{c}(\mathrm{Mpa})$ & $\mathrm{f}_{\mathrm{sp}}(\mathrm{Mpa})$ \\
\hline 156 & 0.00 & 60.72 & 4.32 & 158 & 46.88 & 66.54 & 6.08 \\
\hline 157 & 31.25 & 61.89 & 5.88 & 159 & 31.25 & 29.88 & 3.83 \\
\hline
\end{tabular}

Craig [4]

\begin{tabular}{|c|c|c|c|c|c|c|c|}
\hline no & FRI & $\mathrm{f}^{\prime} \mathrm{c}(\mathrm{Mpa})$ & $\mathrm{f}_{\mathrm{sp}}(\mathrm{Mpa})$ & no & FRI & $\mathrm{f}^{\prime c}(\mathrm{Mpa})$ & $\mathrm{f}_{\mathrm{sp}}(\mathrm{Mpa})$ \\
\hline 160 & 0 & 40.69 & 3.45 & 164 & 120 & 28.97 & 4.55 \\
\hline 161 & 42 & 40.00 & 5.72 & 165 & 200 & 47.59 & 6.00 \\
\hline 162 & 100 & 43.45 & 6.34 & 166 & 120 & 40.00 & 6.07 \\
\hline 163 & 90 & 35.86 & 5.31 & 167 & 160 & 45.52 & 7.10 \\
\hline
\end{tabular}

El-Neima [7] (Comp, splitting, cylinders 150x300mm)

\begin{tabular}{|c|c|c|c|c|c|c|c|}
\hline no & FRI & $\mathrm{f}^{\prime} \mathrm{c}(\mathrm{Mpa})$ & $\mathrm{f}_{\text {sp }}(\mathrm{Mpa})$ & no & FRI & $\mathrm{f}^{\prime} \mathrm{c}(\mathrm{Mpa})$ & $\mathrm{f}_{\text {sp }}(\mathrm{Mpa})$ \\
\hline 168 & 0.00 & 22.34 & 1.96 & 186 & 25.00 & 61.70 & 6.39 \\
\hline 169 & 51.08 & 26.19 & 4.50 & 187 & 25.00 & 39.90 & 5.14 \\
\hline
\end{tabular}




\begin{tabular}{|c|c|c|c|c|c|c|c|}
\hline \multicolumn{8}{|c|}{ (cont) El-Neima [7] } \\
\hline no & FRI & $\mathrm{f}^{\prime} \mathrm{c}(\mathrm{Mpa})$ & $\mathrm{f}_{\mathrm{sp}}(\mathrm{MPa})$ & no & FRI & $\mathrm{f}^{\prime} \mathrm{c}(\mathrm{Mpa})$ & $\mathrm{f}_{\mathrm{sp}}(\mathrm{MPa})$ \\
\hline 170 & 89.39 & 28.57 & 4.60 & 188 & 66.50 & 61.70 & 7.88 \\
\hline 171 & 127.70 & 29.73 & 4.74 & 189 & 133.00 & 67.20 & 10.70 \\
\hline 172 & 38.30 & 24.62 & 3.60 & 190 & 25.00 & 61.70 & 6.39 \\
\hline 173 & 67.03 & 25.24 & 3.88 & 191 & 25.00 & 39.20 & 5.09 \\
\hline 174 & 95.75 & 25.38 & 4.07 & 192 & 66.50 & 61.70 & 7.88 \\
\hline 175 & 25.35 & 23.79 & 3.12 & 193 & 150.00 & 76.70 & 12.11 \\
\hline 176 & 44.37 & 24.76 & 3.64 & 194 & 200.00 & 79.50 & 14.36 \\
\hline 177 & 63.38 & 25.17 & 4.01 & 195 & 250.00 & 77.20 & 16.14 \\
\hline 178 & 25.00 & 61.70 & 6.39 & 196 & 300.00 & 75.80 & 17.98 \\
\hline 179 & 25.00 & 39.90 & 5.14 & 197 & 66.50 & 42.30 & 6.52 \\
\hline 180 & 66.50 & 61.70 & 7.88 & 198 & 100.00 & 41.40 & 7.43 \\
\hline 181 & 133.00 & 67.20 & 10.70 & 199 & 66.50 & 55.70 & 7.48 \\
\hline 182 & 50.00 & 59.30 & 7.14 & 200 & 66.50 & 42.30 & 6.52 \\
\hline 183 & 100.00 & 60.00 & 8.95 & 201 & 133.00 & 71.90 & 11.07 \\
\hline 184 & 150.00 & 67.00 & 11.32 & 202 & 150.00 & 67.00 & 11.32 \\
\hline 185 & 200.00 & 55.90 & 12.04 & & & & \\
\hline \multicolumn{8}{|c|}{ Robert [6] } \\
\hline no & FRI & $\mathrm{f}^{\prime} \mathrm{c}(\mathrm{Mpa})$ & $\mathrm{f}_{\mathrm{sp}}(\mathrm{MPa})$ & no & FRI & $\mathrm{f}^{\prime} \mathrm{c}(\mathrm{Mpa})$ & $\mathrm{f}_{\mathrm{sp}}(\mathrm{MPa})$ \\
\hline 203 & 0.00 & 54.15 & 2.90 & 206 & 42.75 & 56.72 & 5.90 \\
\hline 204 & 14.25 & 55.96 & 5.60 & 207 & 57.00 & 54.15 & 5.60 \\
\hline 205 & 28.50 & 59.47 & 6.00 & 208 & 57.00 & 51.40 & 6.20 \\
\hline \multicolumn{8}{|c|}{ Rjoub and Rasheed [15] } \\
\hline no & FRI & $\mathrm{f}^{\prime} \mathrm{c}(\mathrm{Mpa})$ & $\mathrm{f}_{\mathrm{sp}}(\mathrm{MPa})$ & no & FRI & $\mathrm{f}^{\prime} \mathrm{c}(\mathrm{Mpa})$ & $\mathrm{f}_{\mathrm{sp}}(\mathrm{MPa})$ \\
\hline 209 & 0 & 55.22 & 4.96 & 284 & 0 & 59.76 & 5.18 \\
\hline 210 & 0 & 63.71 & 5.11 & 285 & 0 & 65.65 & 5.26 \\
\hline 211 & 0 & 71.06 & 5.71 & 286 & 0 & 74.69 & 5.91 \\
\hline 212 & 0 & 80.87 & 6.34 & 287 & 0 & 84.84 & 6.74 \\
\hline 213 & 0 & 91.85 & 6.62 & 288 & 0 & 94.78 & 6.77 \\
\hline 214 & 40 & 59.76 & 5.18 & 289 & 40 & 63.07 & 6.1 \\
\hline 215 & 40 & 65.65 & 5.26 & 290 & 40 & 78.66 & 6.79 \\
\hline 216 & 40 & 74.69 & 5.91 & 291 & 40 & 88.3 & 7.36 \\
\hline 217 & 40 & 84.84 & 6.74 & 292 & 40 & 93.94 & 8.81 \\
\hline 218 & 40 & 94.78 & 6.77 & 293 & 40 & 97.06 & 8.95 \\
\hline 219 & 60 & 62.51 & 7.13 & 294 & 60 & 65.21 & 7.32 \\
\hline 220 & 60 & 76.71 & 7.8 & 295 & 60 & 80.42 & 7.95 \\
\hline
\end{tabular}


(cont) Rjoub and Rasheed [15]

\begin{tabular}{|c|c|c|c|c|c|c|c|}
\hline no & FRI & $\mathrm{f}^{\prime} \mathrm{c}(\mathrm{Mpa})$ & $\mathrm{f}_{\mathrm{sp}}(\mathrm{MPa})$ & no & FRI & f'c $(\mathrm{Mpa})$ & $\mathrm{f}_{\mathrm{sp}}(\mathrm{MPa})$ \\
\hline 221 & 60 & 85.62 & 8.62 & 296 & 60 & 90.17 & 8.9 \\
\hline 222 & 60 & 92.98 & 9.64 & 297 & 60 & 94.97 & 9.75 \\
\hline 223 & 60 & 97.03 & 9.83 & 298 & 60 & 98.11 & 9.92 \\
\hline 224 & 80 & 64.73 & 8.12 & 299 & 80 & 67.31 & 8.41 \\
\hline 225 & 80 & 78.91 & 8.93 & 300 & 80 & 81.14 & 9.15 \\
\hline 226 & 80 & 88.01 & 9.69 & 301 & 80 & 92.93 & 10.04 \\
\hline 227 & 80 & 94.67 & 10.96 & 302 & 80 & 96.62 & 11.1 \\
\hline 228 & 80 & 99.21 & 11.47 & 303 & 80 & 99.98 & 11.5 \\
\hline 229 & 120 & 66.81 & 9.22 & 304 & 120 & 69.08 & 9.71 \\
\hline 230 & 120 & 80.82 & 9.98 & 305 & 120 & 82.29 & 10.74 \\
\hline 231 & 120 & 91 & 10.73 & 306 & 120 & 94.17 & 11.23 \\
\hline 232 & 120 & 96.73 & 11.68 & 307 & 120 & 96.35 & 12.04 \\
\hline 233 & 120 & 100.18 & 12.27 & 308 & 120 & 100.2 & 12.63 \\
\hline 234 & 0 & 65.25 & 5.28 & 309 & 0 & 51.22 & 3.95 \\
\hline 235 & 0 & 70.21 & 5.47 & 310 & 0 & 60.85 & 4.1 \\
\hline 236 & 0 & 79.51 & 6.12 & 311 & 0 & 69.39 & 4.42 \\
\hline 237 & 0 & 89.27 & 6.58 & 312 & 0 & 80.11 & 4.75 \\
\hline 238 & 0 & 98.92 & 6.93 & 313 & 0 & 86.48 & 4.98 \\
\hline 239 & 40 & 66.23 & 6.32 & 314 & 40 & 54.71 & 4.94 \\
\hline 240 & 40 & 79.42 & 6.97 & 315 & 40 & 67.91 & 5.94 \\
\hline 241 & 40 & 90.79 & 7.92 & 316 & 40 & 79.31 & 6.28 \\
\hline 242 & 40 & 94.13 & 8.92 & 317 & 40 & 89.76 & 6.63 \\
\hline 243 & 40 & 99.94 & 9.02 & 318 & 40 & 93.62 & 6.91 \\
\hline 244 & 60 & 67.37 & 7.8 & 319 & 60 & 55.84 & 6.41 \\
\hline 245 & 60 & 82.66 & 8.37 & 320 & 60 & 69.42 & 7.04 \\
\hline 246 & 60 & 91.89 & 9.2 & 321 & 60 & 81.61 & 7.68 \\
\hline 247 & 60 & 95.62 & 9.95 & 322 & 60 & 90.31 & 8.2 \\
\hline 248 & 60 & 99.98 & 10.31 & 323 & 60 & 94.28 & 8.57 \\
\hline 249 & 80 & 69.71 & 8.81 & 324 & 80 & 57.88 & 7.23 \\
\hline 250 & 80 & 84.31 & 9.42 & 325 & 80 & 73.07 & 7.92 \\
\hline 251 & 80 & 93.76 & 10.72 & 326 & 80 & 82.60 & 8.45 \\
\hline 252 & 80 & 96.2 & 11.63 & 327 & 80 & 92.61 & 9.57 \\
\hline 253 & 80 & 100.12 & 11.92 & 328 & 80 & 95.31 & 9.92 \\
\hline 254 & 120 & 71.23 & 9.85 & 329 & 120 & 59.12 & 8.21 \\
\hline 255 & 120 & 85.21 & 10.84 & 330 & 120 & 74.24 & 9.00 \\
\hline 256 & 120 & 94.78 & 11.93 & 331 & 120 & 84.63 & 9.64 \\
\hline 257 & 120 & 98.71 & 12.78 & 332 & 120 & 93.11 & 10.36 \\
\hline 258 & 120 & 101.3 & 13.08 & 333 & 120 & 96.72 & 10.74 \\
\hline 259 & 0 & 48.74 & 3.8 & 334 & 0 & 57.87 & 4.08 \\
\hline 260 & 0 & 58.81 & 4.01 & 335 & 0 & 64.08 & 4.25 \\
\hline & & & & & & & \\
\hline
\end{tabular}




\begin{tabular}{|c|c|c|c|c|c|c|c|}
\hline \multicolumn{7}{|c|}{ (cont) Rjoub and Rasheed [15] } \\
\hline No & FRI & f'c (Mpa) & $f_{\text {sp }}(\mathrm{MPa})$ & No & FRI & $f^{\prime}$ c (Mpa) & $f_{\text {sp }}(\mathrm{MPa})$ \\
\hline 261 & 0 & 67.31 & 4.34 & 336 & 0 & 75.31 & 4.63 \\
\hline 262 & 0 & 74.92 & 4.63 & 337 & 120 & 84.42 & 4.82 \\
\hline 263 & 0 & 80.77 & 4.81 & 338 & 120 & 93.64 & 4.15 \\
\hline 264 & 40 & 52.63 & 4.68 & 339 & 120 & 52.63 & 4.68 \\
\hline 265 & 40 & 61.27 & 4.97 & 340 & 0 & 61.72 & 4.97 \\
\hline 266 & 40 & 74.67 & 5.51 & 341 & 0 & 74.67 & 5.51 \\
\hline 267 & 40 & 82.89 & 6.15 & 342 & 0 & 82.89 & 6.15 \\
\hline 268 & 40 & 91.07 & 6.26 & 343 & 40 & 91.07 & 6.26 \\
\hline 269 & 60 & 54.01 & 6.05 & 344 & 60 & 54.01 & 6.05 \\
\hline 270 & 60 & 62.89 & 6.48 & 345 & 60 & 62.89 & 6.48 \\
\hline 271 & 60 & 76.63 & 7.08 & 346 & 60 & 76.63 & 7.08 \\
\hline 272 & 60 & 84.62 & 7.91 & 347 & 60 & 84.62 & 7.91 \\
\hline 273 & 60 & 92.86 & 8.08 & 348 & 60 & 92.82 & 8.08 \\
\hline 274 & 80 & 55.22 & 6.83 & 349 & 80 & 52.2 & 6.83 \\
\hline 275 & 80 & 64.73 & 7.44 & 350 & 80 & 64.73 & 7.44 \\
\hline 276 & 80 & 78.98 & 8.12 & 351 & 80 & 78.98 & 8.12 \\
\hline 277 & 80 & 87.03 & 8.97 & 352 & 80 & 87.03 & 8.97 \\
\hline 278 & 80 & 93.91 & 9.2 & 353 & 80 & 93.91 & 9.2 \\
\hline 279 & 120 & 56.77 & 7.68 & 354 & 120 & 56.77 & 7.68 \\
\hline 280 & 120 & 66.82 & 7.94 & 355 & 120 & 66.82 & 7.94 \\
\hline 281 & 120 & 80.11 & 8.72 & 356 & 120 & 80.11 & 8.72 \\
\hline 282 & 120 & 88.18 & 4.98 & 357 & 120 & 88.18 & 4.98 \\
\hline 283 & 120 & 94.22 & 10.3 & 358 & 120 & 94.22 & 10.33 \\
\hline
\end{tabular}

Note: FRI $=\left(v_{f} * \frac{l}{d}\right) \mathrm{s}$

\section{4- CONCLUSIONS}

The following conclusions can be drawn from this study

1- A suggested expression that predicts the split cylinder tensile strength of steel fiber reinforced concrete is presented.

2- The outcomes of descriptive statistical analysis confirm the credibility of the derived expression.

3- Concrete compressive strength, fiber content and the fiber aspect ratio are the major effectual parameters in specifying the tensile strength of fiber concrete. 


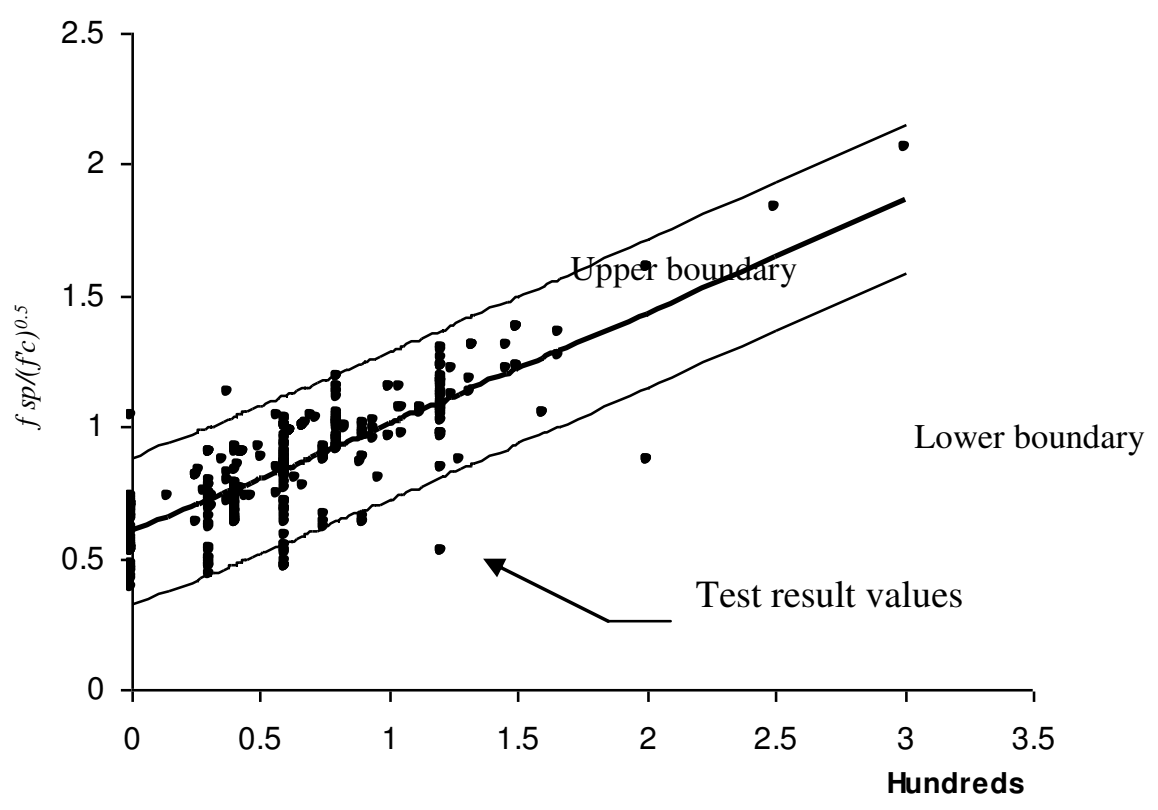

Fiber reinforcement Index,\% FRI

Figure 1: Effect of steel fiber reinforcement index, \% FRI versus $f_{s p} /\left(f_{c}^{\prime}\right)^{0.5}$

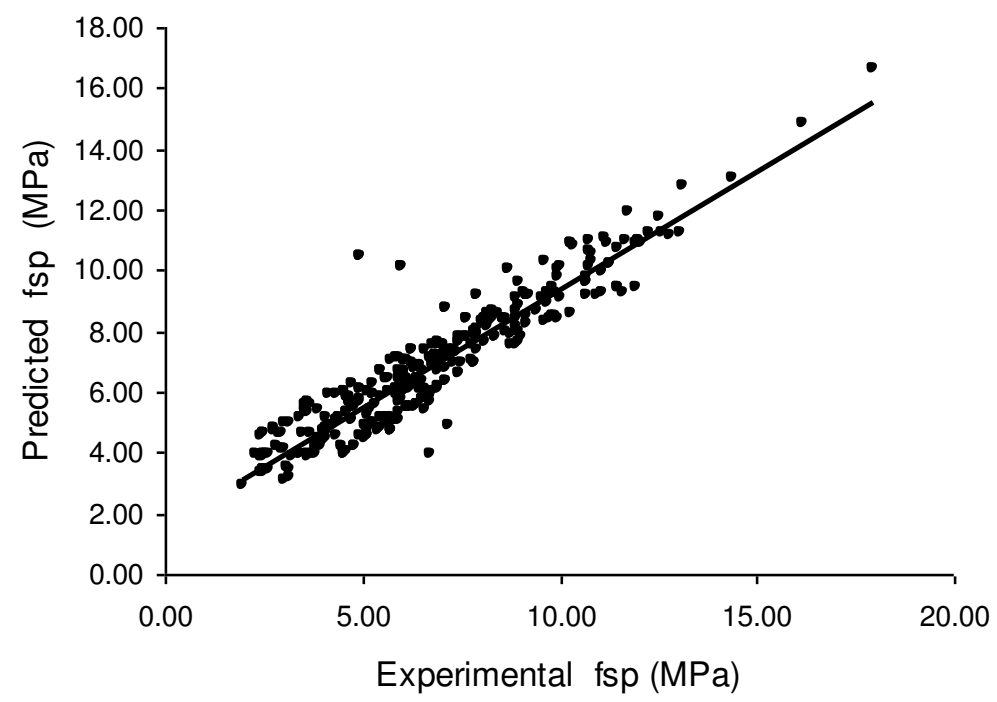

Figure 2: Experimental versus predicted split strength. 


\section{REFERENCES}

[1] Ashour S. A., Hasanian G. S. and Wafa, F. F." Flexural Behavior of High Strength Fiber Reinforced Concrete Beams". ACI Structural Journal, V90, No. 3 May-June 1993, pp279-287.

[2] Ashour S. A. , Hasanian G. S. and Wafa, F. F."Shear Behavior of HighStrength Fiber Reinforced Concrete Beams", ACI Structural Journal, March-April 1992, V. 89, No. 2, pp 176- 184.

[3] Batson, G. Jenkins, E. and Spatney, R. "Steel Fibers as Shear Reinforcement in Beams", ACI Journal, Oct 1972.

[4] Craig, R. J. Parr, J. A. Germain, E. Mosquera, V. Kamilares, S. "Fiber Reinforced Beams in Torsion", ACI Journal, Nov-Dec 1986.

[5] Sharma, A. K., "Shear Strength of Steel Fiber Reinforced Concrete Beams, ACI Journal V. 83, No. 4, 1986, pp. 624-628.

[6] Robert, J. Ward. and Victor C. Li. " Dependence of Flexural Behavior of Fiber Reinforced Mortar on Material Fracture Resistance and Beam Size", ACI Materials Journal, Nov-Dec 1990, V87, No.6, pp627-637.

[7] EL-Niema, E. I. " Reinforced Concrete Beams with steel Fibers under Shear", ACI Structural Journal, March-April 1991, V. 88, No. 2, pp 178183.

[8] Ashour S. A. , Hasanian G. S. and Wafa, F. F." Flexural Behavior of High Strength Fiber Reinforced Concrete Beams". ACI Structural Journal, V90, No. 3 May-June 1993, pp279-287

[9] Ghosheh, N. "Reinforced Concrete Beams with steel Fibers ", M. Sc. Thesis, Jordan University, 1999.

[10] Padmarajaiah, S. K.and Ramaswamy, A. " Crack-Width Prediction for High-Strength Concrete Fully and Partially Prestressed Beam Specimens Containing Stee Fibers", ACI Structural Journal, November-December 2001, V. 98, no.6, pp852-861

[11] Marar K.and Celik, T. "The Influence of FRI on the Relationship Between Compressive and Tensile Strength of NSFRC and HSFRC" The sixth International Conference on Concrete Technology, Amman-Jordan, Oct. 2002.

[12] Kwak, Y. K. Eberhard, M. O. Kim, W. S. and Kim, J. "Shear Strength of Steel Fiber-Reinforced Concrete Beams without Stirrups", ACI Structural Journal, July-August 2002, V.99, No. 4, pp530-538. 
[13] Ayish, M. "Punching Shear Behavior of Flat Plates with Fiber Reinforced Concrete", M. Sc. Thesis, June 2004, Jordan University of Sceince and Technology.

[14] Bani-Yasin, I. S. "Performance of High Strength Fibrous Concrete slab concrete connections under gravity and lateral loads" M. Sc. thesis, Jordan University of Sceince and technology", June, 2004.

[15] Rjoub, M. I. M., Rasheed, T.M. "Shear Strength of Steel Fiber High Strength Concrete Beams", Seventh International Conference on Concrete Technology, Oct. 2004.

[16] Aqaileh, Z. A. "The structural Behavior of High Strength Normal Two Way Ribbed Slabs with Steel fibers", M. Sc. Thesis, Jordan University, 2004.

\section{حساب مقاومة شد الانفلاق للخرسانة بالألياف}

أصبح استخدام الألياف المعدنيـة بالخرسـانة بتزايد مستمر في الوقت الحسالي، و ذلك بسبب تحسن

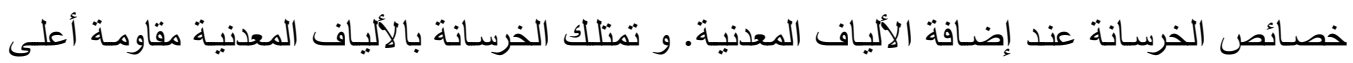

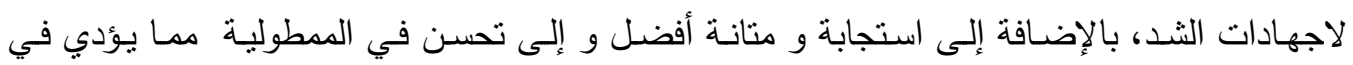

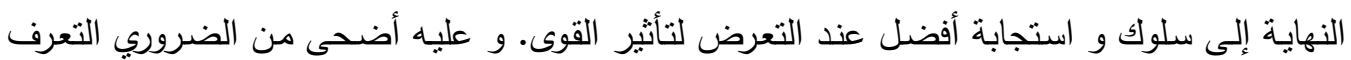

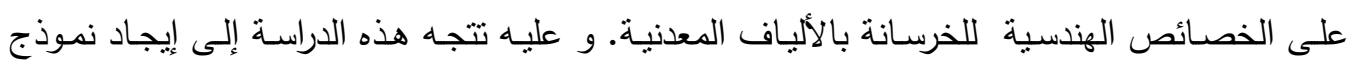

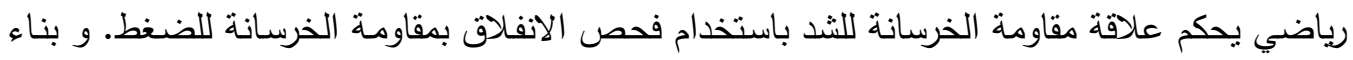

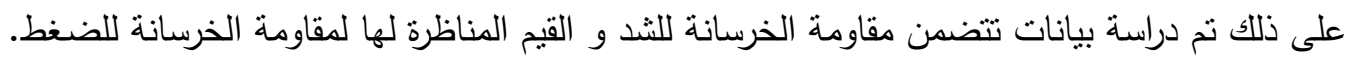

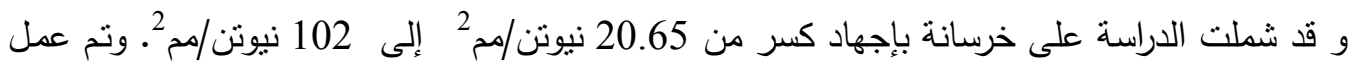

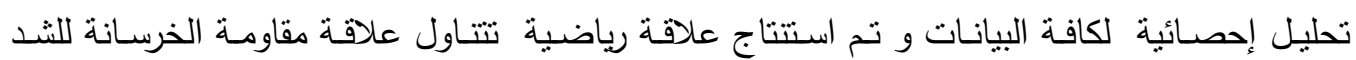
باستخدام فحص الانفلاق بمقاومة الخرسانة للضغط لنفس الخلطات.

الباحث الأول: الدكتور مازن علي مسمار : كلية الهندة التكنولوجية| قسم الهندسة المدنيةا جامعة البلقاء التطبيقية| عمان \1134 ص.ب. 15008 \الأردن الأن الباحث الثاني: الدكتور محدد مذيب الرجوب: كلية الهندسة التكنولوجيةا قسم الهندسة الددنية| جامعة البلقاء النطبيقية| عمان \} 1 1 3 4 \text { ص.ب. } 1 5 0 0 8 \text { \الأردن } 